\title{
New Elliptic System and Global Solutions for the Constraints Equations in General Relativity
}

\author{
YvonNe ChOQuet-BRuHAT \\ Département de Mécanique, Université de Paris, Paris, France
}

Received November 1, 1970

\begin{abstract}
By a new choice of the arbitrarily given quantities on an initial 3-manifold we reduce the system of constraints, in General Relativity, to an elliptic system of four equations, the coefficients of which have a simple geometric interpretation on the 3-manifold. The system seems well suited for a global study and some results are given in this direction.
\end{abstract}

\section{Introduction}

An initial data set $\mathscr{I}$, in General Relativity ${ }^{1}$, is a 3 dimensional differentiable manifold $V_{3}$, a negative definite riemannian metric on $V_{3}, \overline{\boldsymbol{g}}$, and a second rank, symmetric, tensor $\boldsymbol{P}$, satisfying the constraint equations:

$$
\begin{array}{ll}
\bar{\nabla}_{j}\left(P_{i}^{j}-\delta_{i}^{j} P\right)=0, & P=P_{i}^{i}, \\
\bar{R}+H^{2}-P^{2}=0, & H^{2}=P_{i j} P^{i j}
\end{array}
$$

Where $\bar{R}$ is the riemannian scalar curvature, and $\bar{\nabla}$ the covariant derivative of $\overline{\boldsymbol{g}}$.

An einsteinian space time is a 4-dimensional differentiable manifold $V_{4}$, endowed with a hyperbolic metric $\boldsymbol{g}$, with vanishing Ricci tensor. It is said that $\left(V_{4}, \boldsymbol{g}\right)$ is a solution of the Cauchy problem, associated with the initial data $\mathscr{I}$, if there is a diffeomorphism $\Lambda$ of $V_{3}$ with a submanifold $\Sigma$ of $V_{4}$ such that the image by $\Lambda$ of $\overline{\boldsymbol{g}}$ and $\boldsymbol{P}$ coinncide respectively with the metric induced on $\Sigma$ by $\Lambda$ and the second fundamental form of $\Sigma$ as submanifold of $\left(V_{4}, \boldsymbol{g}\right)$.

It is known that to each initial data set corresponds an einsteinian space time, and only one in the class of maximal, globally hyperbolic space times [4].

The system of constraint equations (1), (2) has been already extensively studied ${ }^{2}$, but rather few global (i.e. interesting) solutions are

${ }^{1}$ We speak, for simplicity, of empty-space equations. We will, in an appendix, show how the interior case can be treated along the same lines.

${ }^{2}$ For a bibliography up to 1962 see Bruhat (1962). 
known. The form (1), (2) does not seem convenient for a global mathematical study. Starting from the idea of Wheeler and Sharp of the "thin sandwich" formulation of constraints (important from the quantization point of view), and using harmonic coordinates, I have written (Bruhat, 1962) the constraints as an elliptic system for the tensorial densities $G^{00}, G^{0 i}$, the $G^{i j}$ and $\partial_{0} G^{i j}$ being given arbitrarily (with $G^{i j}$ negative definite).

Using involved techniques and the Schauder fixed point theorem, it has been proved (Vaillant, 1967) that this elliptic system has a global solution (with $G^{00}>0$ ) on a manifold $V_{3}$ homeomorphic to $R^{3}$, if $G^{i j}$ falls off exponentially to the euclidean metric at infinity and $\partial_{0} G^{i j}$ is small enough.

A drawback of the obtained system is that it is coordinate dependant, and is not suitable for the study on a manifold non homeomorphic to $R^{3}$. More over its coefficients have no simple geometric meaning ${ }^{3}$. We will therefore, in this paper, take another line of approach, which originates from the first work on constraints by Lichnerowicz (1944) and has had already several applications in a particular case, the so called "static" case.

In his 1944 paper Lichnerowicz shows that if:

then

$$
\bar{d} s^{2}=\varphi^{4} d s^{* 2}
$$

$$
\bar{R} \equiv \frac{1}{\varphi^{5}}\left(-8 \Delta^{*} \varphi+R^{*} \varphi\right)
$$

where $R^{*}$ and $\Delta^{*}$ are the scalar curvature and Laplacian of the metric $g^{*}$. The Eq. (2) then reads:

$$
-8 \Delta^{*} \varphi+R^{*} \varphi+L \varphi^{5}=0
$$

which is a rather simple equation if $L$ is given (particularly if $L=0$ ). We will in this paper write the three remaining equations under a form such that their principal part will also be the Laplacian $\Delta^{*}$ of an unknown vector $\lambda_{i}^{*}$.

We will then give some classes of obvious solutions of the obtained equations.

\section{A. Elliptic System}

\section{Some Formulas in an Orthonormal Moving Frame}

We will, in order to have in evidence geometrically meaningful quantities, use the Cartan's method of orthonormal tetrads. Let

$$
d s^{2}=g_{\alpha \beta} d x^{\alpha} d x^{\beta}=\left(V d x^{0}\right)^{2}+\sum_{i=1}^{3}\left(a_{i j} d x^{j}+\lambda_{i} d x^{0}\right)^{2}
$$

${ }^{3}$ On the other hand it has been proved (Komar, 1968) that the system obtained by giving arbitrarily $g_{i j}$ and $\partial_{0} g_{i j}$ is not of classical type, and unstable. 
be an expression of the space-time metric $g$ in an orthonormal frame. The quantities in the right of (1.1) are related to the metric $g_{\alpha \beta}$ in the natural frame by:

$$
g_{00}=V^{2}+\sum_{i=1}^{3} \lambda_{i}^{2}, \quad \sum_{i=1}^{3} a_{i j} a_{i h}=g_{j h}=\bar{g}_{j h}, \quad \sum_{i=1}^{3} \lambda_{i} a_{i j}=g_{j 0}
$$

where $\overline{\boldsymbol{g}}$ is the metric induced on $S\left(x^{0}=0\right)$ by $\boldsymbol{g}$.

We have $V$ real, $\lambda_{i}$ and $a_{i j}$ purely imaginary. If we denote by $a^{i j}$ the inverse matrix of $a_{i j}$, and $\bar{g}^{j h}$ the inverse matrix of $g_{j h}$ we have:

$$
a^{i j} a^{i h}=\bar{g}^{j h} .
$$

The extrinsic curvature of $S\left(x^{0}=0\right)$ is given by (cf. Fourès-Bruhat, 1956):

$$
P_{i j}=\frac{\bar{\nabla}_{i} \lambda_{j}+\bar{\nabla}_{j} \lambda_{i}}{2 V}-\frac{1}{2 V}\left(a^{i h} \frac{\partial a_{j h}}{\partial x^{0}}+a^{j h} \frac{\partial a_{i h}}{d x^{0}}\right)
$$

where $\bar{\nabla}$ is the covariant derivative in the metric $\bar{g}$.

\section{Initial Metric Conformal to a Given Metric}

Suppose that the metric $\overline{\boldsymbol{g}}$ of $S$ is given up to a conformal scalar factor which we call for further convenience $\varphi^{4}$; thus, in the orthonormal frame:

$$
a_{i j}=\varphi^{2} a_{i j}^{*}, \quad a_{i j}^{*} a_{i h}^{*}=g_{j h}^{*}
$$

where $g^{*}$ is the given metric on $S$.

We define $\lambda_{i}^{*}$ by:

$$
\lambda_{i}=\varphi^{2} \lambda_{i}^{*}
$$

and $A_{i j}^{*}$ to be the symetric tensor on $S$ :

$$
A_{i j}^{*}=a^{* i h} \frac{\partial a_{j h}^{*}}{\partial x^{0}}+a^{* j h} \frac{\partial a_{i h}^{*}}{\partial x^{0}} .
$$

The coefficients of connexion $\gamma_{i j k}=\bar{\gamma}_{i j k}$ and $\gamma_{i j k}^{*}$, related to the metrics $\overline{\boldsymbol{g}}$ and $g^{*}$ respectively, are easily found (cf. [2]) to be such that

$$
\bar{\gamma}_{i j h}=\frac{1}{\varphi^{2}}\left\{\gamma_{i j h}^{*}+\frac{2 \nabla_{i}^{*} \varphi}{\varphi} \delta_{j h}-\frac{2 \nabla_{j}^{*} \varphi}{\varphi} \delta_{i h}\right\}
$$

while the derivative $\nabla_{i}^{*} \varphi$ of a scalar $\varphi$ is given by

$$
\partial_{i}^{*} \varphi=\nabla_{i}^{*} \varphi=\frac{1}{\varphi^{2}} \bar{\nabla}_{i} \varphi=\frac{1}{\varphi^{2}} \partial_{i} \varphi .
$$

The covariant derivative of a vector being:

$$
\bar{\nabla}_{j} \lambda_{i}=\partial_{j} \lambda_{i}+\bar{\gamma}_{k i j} \lambda_{k} \text {. }
$$


A straight forward computation shows then that:

$$
P_{i j}=\frac{1}{2 V}\left(\nabla_{i}^{*} \lambda_{j}^{*}+\nabla_{j}^{*} \lambda_{i}^{*}\right)+\frac{2}{\varphi V} \lambda_{k}^{*} \nabla_{k}^{*} \varphi \delta_{i j}-\frac{1}{2 V} A_{i j}^{*}-\frac{2}{\varphi V} \delta_{i j} \frac{\partial \varphi}{\partial x^{0}}
$$

$P$, the mean extrinsic curvature of $S$, being then

with

$$
P=\frac{1}{V}\left(\nabla_{i}^{*} \lambda_{i}^{*}\right)+\frac{6}{\varphi V} \lambda_{k}^{*} \nabla_{k}^{*} \varphi-\frac{1}{2 V} A^{*}-\frac{6}{\varphi V} \frac{\partial \varphi}{\partial x^{0}}
$$

$$
A^{*}=\sum_{i=1}^{3} A_{i i}^{*}=\frac{\partial}{\partial x^{0}} \log \left|a^{*}\right|^{2}, \quad a^{*}=\operatorname{det}\left(a_{i j}^{*}\right) .
$$

They are:

\section{Constraint Equations}

$$
\begin{gathered}
-S_{0 i} \equiv \overline{\nabla_{j}} P_{i j}-\overline{\nabla_{i}} P=0, \\
-2 S_{00} \equiv \bar{R}+H^{2}-P^{2}=0 .
\end{gathered}
$$

It is already known that 3.2 gives:

$$
-8 \Delta^{*} \varphi+R^{*} \varphi+L \varphi^{5}=0, \quad L=P_{i j} P_{i j}-P^{2} .
$$

Using the Ricci identity, and denoting by $\Delta^{*} \lambda_{i}^{*}$ a generalized Laplacian for the vector $\lambda_{i}^{*}$ (i.e. $\Delta^{*} \lambda_{i}^{*}=\nabla_{j}^{*} \nabla_{j}^{*} \lambda_{i}^{*}-R_{i j}^{*} \lambda_{j}^{*}$ ) we get:

$$
\begin{aligned}
S_{0 i}= & -\frac{1}{2 V} \Delta^{*} \lambda_{i}^{*}+4 \nabla_{i}^{*}\left(\lambda_{k}^{*} \frac{\partial_{k}^{*} \varphi}{\varphi V}\right) \\
& +\frac{1}{2 V^{2}}\left(\nabla_{i}^{*} \lambda_{j}^{*}+\nabla_{j}^{*} \lambda_{i}^{*}\right) \nabla_{j}^{*} V-\frac{1}{2 V^{2}}\left(\nabla_{j}^{*} \lambda_{j}^{*}\right) \nabla_{i}^{*} V . \\
& +\nabla_{i}^{*}\left(\frac{\nabla_{j}^{*} \lambda_{j}^{*}}{2 V}-\frac{4}{\varphi V} \frac{\partial \varphi}{\partial x^{0}}\right)-\frac{1}{\varphi^{2}}\left\{\frac{6 P_{i k} \partial_{k}^{*} \varphi}{\varphi}-\frac{2 P \nabla_{i}^{*} \varphi}{\varphi}\right\} \\
& +\nabla_{j}^{*}\left(A_{i j}^{*}-\delta_{i j} A^{*}\right)=0 .
\end{aligned}
$$

The Eqs. (3.4) simplify by the gauge conditions:

and (less essential)

$$
\frac{1}{\varphi} \frac{\partial \varphi}{\partial x^{0}}=\frac{1}{8} \nabla_{j}^{*} \lambda_{j}^{*}
$$

$$
V=1
$$

to the equations where the principal part in $\lambda_{i}^{*}$ is $\Delta^{*} \lambda_{i}^{*}$ :

$$
S_{0 i} \varphi^{2}=-\Delta^{*} \lambda_{i}+8 \nabla_{i}^{*}\left(\lambda_{k}^{*} \frac{\partial_{k} \varphi}{\varphi}\right)-\frac{1}{\varphi}\left(6 P_{i k} \partial_{k}^{*} \varphi-2 P \partial_{i}^{*} \varphi\right)+\nabla_{j}^{*} \alpha_{i j}^{*}=0
$$

where $\alpha_{i j}^{*}=\frac{1}{2}\left(A_{i j}^{*}-\delta_{i j} A^{*}\right)$ is an arbitrary symmetric tensor. 
Let us remark that the gauge conditions (3.5) and (3.6) do not restrict the generality of the solutions of the constraints, since, for an arbitrary space time, we can always find orthonormal frames and conformal metric $g^{*}$ on $S$ such that $\lambda_{i}=0$ and $V=\varphi=1$.

We can now recapitulate the obtained results under the form of a theorem:

Theorem: The most general initial data set is obtained by

1) giving arbitrarily on a 3 dimensional manifold $V_{3}$ a metric $g^{*}$, negative definite and a second rank symmetric tensor $A_{i j}^{*}$.

2) Solving on $V_{3}$ the elliptic system 3.3,3.7 for the unknown $\lambda_{i}^{*}, \varphi$. The geometric initial datas $\bar{g}$ and $P$ being then given by

$$
\begin{gathered}
\bar{g}=\varphi^{4} g^{*}, \\
P_{i j}=\frac{1}{2}\left(\nabla_{i}^{*} \lambda_{j}^{*}+\nabla_{j}^{*} \lambda_{i}^{*}\right)-\frac{1}{4}\left(\nabla_{k}^{*} \lambda_{k}^{*}\right) \delta_{i j}+\frac{2}{\varphi} \lambda_{k}^{*} \nabla_{k}^{*} \varphi \delta_{i j}-\frac{1}{2} A_{i j}^{*} .
\end{gathered}
$$

\section{Ellipticity}

The characteristic determinant of the system of partial differential equations (3.3) and (3.7), for the four unknown $\varphi, \lambda_{i}^{*}$ is:

$$
\left(-g_{i j}^{*} X^{i} X^{j}\right)^{4}
$$

and thus, $g^{*}$ being negative definite, this system is elliptic on $V^{3}$. These equations have a linear principal part, $-\Delta^{*}$, but are non linear. The methods developped by Vaillant-Simon in [5] to construct solutions of an analogous system (which is not strongly elliptic, due to the term $\nabla_{i}^{*}\left(\lambda_{k} \frac{\partial_{k}^{*} \varphi}{\varphi}\right)$ in (3.7)) on $V_{3}$ homeomorphic to $R^{3}$, with $g^{*}$ asymptotically euclidean (with an exponential fall off), will probably apply here. On the other hand the form of the Eqs. (3.3) and (3.7), manifestly covariant on $V_{3}$, seems well appropriate for solutions on a general manifold $V_{3}$.

\section{B. Some Classes of Solutions}

We will give here some global solutions, on a 3. dimensional manifold $V_{3}$; of the constraints $3.3,3.7$ by making, first, a convenient choice for the arbitrarily given quantities.

\section{Time Symmetric (or Static) Case}

The three Eqs. (3.7) are obviously satisfied by the choice

$$
A_{i j}^{*}=0, \quad \lambda_{i}^{*}=0
$$


which is, by definition, the "time symmetric" case $\left(g_{0 i}=0, \frac{\partial g_{i j}}{\partial x^{0}}=0\right)$ the last equation reduces then to the well known one:

$$
-\Delta^{*} \varphi+\frac{1}{8} R^{*} \varphi=0
$$

which has (Araki, 1959) at most one admissible solution (i.e. $\varphi>0$ and bounded). The solutions exists on $V_{3}=R_{3}$ if $g^{*}$ is asymptotically euclidean i.e. euclidean outside a compact (Araki, 1959) or exponentialy falling off to euclidean (Simon-Vaillant, 1965).

One can study the Eq. (5.1) on a manifold $V_{3}$ by Hilbert space methods.

It is easy to prove that if $g^{*}$ and $R^{*}$ are locally integrable and bounded on $V_{3}$ with $R^{*}<0$, and $R^{*}$ square integrable on $V_{3}$ then the equation (deduced from (5.1) by setting $\varphi=1+u$ )

$$
-\Delta^{*} u+\frac{1}{8} R^{*} u=-\frac{1}{8} R^{*}
$$

has one and only one solution (in a weak sense) $u \in H_{0}^{1}\left(V_{3}\right)$, where $H_{0}^{1}\left(V_{3}\right)$ is the closure in $H^{1}\left(V_{3}\right)$ of $\mathscr{D}$ (infinitely differentiable functions with compact support), where $H^{1}\left(V_{3}\right)$ is the Hilbert space of functions on $V_{3}$ which are square integrable together with their first derivatives in the distribution sense. Thus we have two kinds of results:

1) $V_{3}$ is compact: then the function $u=-1$ on $V_{3}$ belongs to $H_{0}^{1}\left(V_{3}\right)=H^{1}\left(V_{3}\right)$, and the only, non physically admissible, solution of (5.2) (with $\varphi-1 \in H^{1}\left(V_{3}\right)$ ) is $\varphi=0$

2) $V_{3}$ is non compact then, in general, $u=-1$ does not belong to $H_{0}^{1}\left(V_{3}\right)$. The solution $\varphi=1+u, u \in H_{0}^{1}\left(V_{3}\right)$ may be physically admissible. It has been shown (Vaillant, 1965), by use in particular of the maximum principle, that $\varphi$ is of class $C^{2}$, and $\varphi>0$ if $V_{3}=R^{3}$ and if $g^{*}$ is of class $C^{2}$.

\section{Conformally Minimal Initial Surface}

It is still possible to satisfy the Eqs. (3.7) by taking $\lambda_{i}^{*}=0$ if the arbitrary quantities $a_{i j}^{*}, \frac{\partial a_{i j}^{*}}{\partial x^{0}}$ and the unknown $\varphi$ satisfy the following relations (which make the Eqs. (3.7) homogeneous in $\lambda_{i}^{*}$ 's):

$$
\frac{1}{\varphi}\left(3 A_{i k}^{*} \partial_{k}^{*} \varphi-A^{*} \partial_{i}^{*} \varphi\right)+\frac{1}{2} \nabla_{j}^{*}\left(A_{i j}^{*}-\delta_{i j} A^{*}\right)=0
$$

which may be written:

$$
\frac{1}{2 \varphi^{6}} \nabla_{j}^{*}\left(A_{i j}^{*} \varphi^{6}\right)-\frac{1}{\varphi^{2}} \nabla_{i}^{*}\left(A^{*} \varphi^{2}\right)=0 .
$$


Instead of giving arbitrarily the second rank tensor $\frac{\partial \varphi_{i k}^{*}}{\partial x^{0}}$ we will consider as given the tensor

$$
B_{i j}^{*}=A_{i j}^{*} \varphi^{6}
$$

and will choose it so that the relation (6.2) is satisfied. This may be done by imposing on the arbitrary tensor $B_{i j}^{*}$ the properties

and

$$
\nabla_{j}^{*} B_{i j}^{*}=0
$$

$$
B^{*}=0 \text {. }
$$

The conditions $B^{*}=0$ expresses (when $\lambda_{i}^{*}=0$ ) the fact that the initial manifold $S$ is a minimal surface ${ }^{4}$ of the space-time.

$B_{i j}^{*}$ being given satisfying (6.3) and (6.4) and $\lambda_{i}^{*}=0$ taken as solution of (3.7) (which is the only one with reasonnable behavior), the only equation remaining to solve is (3.3) wich reads now:

$$
-8 \Delta^{*} \varphi+R^{*} \varphi+L^{*} \varphi^{-7}=0
$$

where we have set

$$
L^{*}=\frac{1}{4} B_{i j}^{*} B_{i j}^{*}>0
$$

The standard methods on monotone operators do not apply here, since $\varphi^{-7}$ is not defined on a vector space. However it is certainly possible to construct, in special cases, solutions of the Eq. (6.5) on manifolds (a solution in $R^{3}$, for $R^{*}<0$ and $L^{*}$ small enough is easy to obtain, it has already been given, in a bounded domain of $R^{3}$, in Lichnerowicz [1]).

\section{Appendix}

\section{Interior Case}

When the stress-energy tensor $T_{\alpha \beta}$ does not vanish Eqs. (3.7) and (3.3) become:

$$
\begin{gathered}
-\Delta^{*} \lambda_{i}+8 \nabla_{i}^{*}\left(\lambda_{k}^{*} \frac{\partial_{k} \varphi}{\varphi}\right)-\frac{1}{\varphi}\left(6 P_{i k} \partial_{k}^{*} \varphi-2 P \partial_{i}^{*} \varphi\right)+\nabla_{j}^{*} \alpha_{i j}^{*}=\varphi^{2} T_{0 i} \\
-8 \Delta^{*} \varphi+R^{*} \varphi+L \varphi^{5}+T_{00} \varphi^{5}=0
\end{gathered}
$$

where, by physical arguments $T_{00}>0$.

When $T_{0 i}=0$ the methods used in $\S 5$ and 6 to construct global solutions (with $\lambda_{i}=0$ ) may still be used.

\footnotetext{
${ }^{4}$ Cf. Lichnerowicz [1] who also used this property in a somewhat different context.
} 
Let us consider, for example, the case where $T_{\alpha \beta}$ is the stress-energy tensor of a one particle distribution function $f(x, p)$, then:

$$
T_{\alpha \beta}(x)=\int_{P_{x}} f(x, p) p_{\alpha} p_{\beta} \bar{\omega}
$$

where $P_{x}$ is the mass-hyperboloïd at $x$

$$
g_{\alpha \beta}(x) p^{\alpha} p^{\beta}=m^{2} \quad p^{\alpha}>0
$$

and $\bar{\omega}$ the invariant volume element of $P_{x}$ :

$$
\bar{\omega}=\frac{\bar{\eta}}{p_{0}}, \quad \bar{\eta}=|g|^{\frac{1}{2}} d p^{1} \cap d p^{2} \cap d p^{3}
$$

let us denote by $\bar{f}\left(x^{i}, \Pi_{i}\right)$ the expression of $f(x, p)$ for $x \in S$, when the $\Pi_{i}$ are the space components (in the orthonormal frame) (1.1) of the vector $p$ (momentum of the particle). We have, on $S$

$$
T_{0 i}=\int_{P_{x}} \bar{f}\left(x^{j}, \Pi_{j}\right) \Pi_{i} \bar{\eta}=\frac{1}{2} \int_{P_{x}}\left\{\bar{f}\left(x^{j} \Pi_{j}\right)+\bar{f}\left(x^{j}-\Pi_{j}\right)\right\} \Pi_{i} \bar{\eta}
$$

which gives the sufficient condition for $T_{0 i}=0$

$$
\bar{f}\left(x^{j}, \Pi_{j}\right)=-\bar{f}\left(x^{j},-\Pi_{j}\right) .
$$

\title{
References
}

1. Lichnerowicz, A.: J. Math. Pures Appl. 23, 37-63 (1944).

2. Fourès-Bruhat, Y.: J. Rat. Mech. Anal. 5, 951—-966 (1956).

3. Bruhat, Y.: In: Gravitation, L. Witten ed. New York: J. Wiley 1962.

4. Choquet-Bruhat, Y., Geroch, R.: Commun. math. Phys. 14, 329-335 (1969).

5. Vaillant, A.: J. Math. Pures Appl. 48, 1-90 (1969).

6. - Thèse de 3e cycle Paris 1965 (mimeographed).

\author{
Y. Choquet-Bruhat \\ Département de Mécanique \\ Université de Paris \\ Tours 65-66-11. Quai St. Bernard \\ F-75 Paris 5/France
}

